Ann. Zootech., I972, 21 (2), 233-243.

\title{
ÉTUDE DE DIFFÉRENTES TECHNIQUES D'ALIMENTATION EN VUE DE LA PRODUCTION D'UN POULET DE CHAIR DE QUALITÉ
}

\author{
J. SIMON et P. DELPECH \\ Station de Recherches avicoles, \\ Centre de Recherches de Tours, I. N. R. A., \\ 37 - Nouzilly \\ Laboratoire de la chaire de Zootechnie \\ École nationale supérieure agronomique, I.N.R.A., \\ 78 - Thiverval Grignon
}

\section{RÉSUMÉ}

Afin de produire un poulet de qualité, nous cherchons à définir des méthodes de rationnement qui freinent le développement pondéral et permettent d'atteindre un poids vif de $2 \mathrm{~kg}$ à I 2 semaines sans altérer l'apparence et la composition corporelle.

Dans cet essai réalisé avec des poussins mâles à croissance rapide Arbor Acres, l'alimentation est toujours libre. Trois programmes alimentaires sont comparés; ils sont définis en cours d'expérience en fonction de la croissance des animaux. Dans un de ces programmes (Lot D), les animaux reçoivent successivement : de 0 à 6 semaines un régime de démarrage $(2 \mathrm{r}, 6 \mathrm{p}$. Ioo de protéines), puis de 6 à 9 semaines un régime de finition (I6,6 p. Ioo de protéines) et pendant les trois dernières semaines un mélange de céréales. Dans un autre cas (Lot F), les animaux reçoivent dès la naissance le régime finition, puis à l'âge de to semaines le mélange de céréales. Selon le dernier programme, les animaux reçoivent alternativement dès leur naissance le mélange de céréales puis le régime de démarrage. Deux périodes sont étudiées : soit 3 jours de céréales puis 4 jours de régime démarrage (Lot $3 \mathrm{C}_{-4} \mathrm{D}$ ), soit 7 jours de céréales puis 7 jours de régime démarrage (Lot $7 \mathrm{C}-7 \mathrm{D}$ ).

Pour les critères suivants : consommation et efficacité alimentaires, engraissement et coût de l'alimentation, la distribution du régime finition dès la naissance constitue la technique la plus défavorable alors que la restriction périodique donne les meilleurs résultats. L'alimentation alternée apparaît donc comme une méthode intéressante pour la production de poulets de qualité répondant aux exigences des consommateurs.

\section{INTRODUCTION}

La production d'un poulet de chair de qualité, répondant aux exigences des consommateurs français, impose de sacrifier des animaux âgés d'au moins I2 semaines, ne pesant pas plus de $2 \mathrm{~kg}$ (DEL,PECH, I963, I968, I969). Avec les souches 
modernes, cet objectif ne peut être atteint qu'en empêchant l'animal de se développer normalement. Le but de l'essai est de préciser la méthode de rationnement qui assure cette performance au moindre côtt tout en conservant aux carcasses la composition corporelle et l'aspect qui plaisent au consommateur.

Classiquement, le ralentissement de la croissance du poulet est effectué par la distribution de céréales en fin d'élevage et par l'utilisation de souches à croissance relativement lente. Une autre modalité consiste à distribuer aux sujets dès leur naissance un régime moins concentré en énergie et protéines. Enfin, des études récentes ont montré que le Poulet s'adapte à des restrictions alimentaires intermittentes (Simon, Blum et Jacquot, ig68 ; Simon et Blum, sous presse). En effet, les restrictions intervenant périodiquement induisent une hyperphagie adaptative et améliorent temporairement la vitesse de croissance et l'efficacité alimentaire. Ces modifications permettent au poulet d'effacer partiellement et avec un bon rendement l'effet néfaste des restrictions successives. Il paraît donc possible de ralentir la croissance en remplaçant périodiquement un régime riche et équilibré par un mélange de céréales. Un tel mode d'alimentation revient à effectuer un rationnement qualitatif discontinu. Ce programme aurait l'avantage d'utiliser des céréales qui sont en général disponibles chez l'éleveur et surtout de bénéficier de l'adaptation à l'alimentation discontinue. Le ralentissement se ferait alors au moindre coût. Au cours de cette expérience, nous comparons les trois modalités de restrictions alimentaires et étudions leurs effets sur la croissance, 1'état d'engraissement et le coût de l'alimentation, l'objectif étant d'amener les animaux à un même poids de $2 \mathrm{~kg}$ à I2 semaines par ces différentes voies.

\title{
MATÉRIEL ETT MÉTHODES
}

\author{
Régimes
}

Trois régimes sont distribués aux animaux selon des modalités différentes exposées plus loin. Leur composition est indiquée dans le tableau $x$. Le premier correspond à un régime " démarrage ", le deuxième à un régime "finition ", le troisième est un mélange de céréales broyées sans adjonction de complément minéral ou vitaminique. Ces rations sont en farine et distribuées à volonté. Les régimes sont isoénergétiques d'après le calcul $295 \circ$ à 2975 Kcalories d'énergie métabolisable $/ \mathrm{kg}$ de régime) et apportent respectivement $2 \mathrm{I}, 6, \mathrm{I} 6,6$ et 10,5 p. Ioo de protéines brutes. Un anticoccidien (Amprolium) est distribué dans l'eau de boisson aux doses habituelles. Tous les régimes sont distribués à volonté.

\section{Animaux-lots}

384 poussins mâles $A r b o r-A c r e s$ sont élevés au sol et éclairés pendant I 4 heures par jour. L'expérience débute dès la naissance et dure 12 semaines. Les animaux sont répartis au hasard, par tirage au sort, en 4 lots. Pour chaque lot, il existe 6 répétitions de 16 individus. Selon les lots, des restrictions alimentaires sont imposées, maintenues, intensifiées ou, au contraire, levées pour permettre aux poulets d'atteindre en fin d'expérience le poids vif fixé. Elles s'exercent par l'intermédiaire de changements dans la qualité des régimes. Le lot (D) est restreint tardivement; il reçoit successivement le régime démarrage, le régime finition et enfin le mélange de céréales. Le lot $(F)$ qui est freiné dès la naissance est alimenté d'abord avec le régime de type finition, puis avec le mélange de céréales. En fin deux lots reçoivent en alternance le mélange de céréales (C) et le régime démarrage (D). Le lot $3 \mathrm{C}-4 \mathrm{D}$ se caractérise par une période de 7 jours au cours de laquelle alternent 3 jours de céréales et 4 jours de démarrage. Le lot 7 C-7 D 
avec une période de $\mathbf{I} 4$ jours, reçoit pendant 7 jours consécutifs et successivement : le mélange de céréales puis le régime démarrage. Ainsi, pour les deux lots soumis à l' " alimentation discontinue ", la restriction est intermittente et intervient dès le jeune âge. Dans tous les lots, les plans d'alimentation précis, c'est-à-dire les époques de changement de régime, sont déterminés d'après l'évolution pondérale des animaux. Les schémas définitifs apparaissent dans les résultats.

TABLEAU I

Composition des régimes (p. Io0)

\begin{tabular}{|c|c|c|c|c|c|}
\hline Démarrage (D) & P. 100 & Finition $(F)$ & P. 100 & Mélange de Céréales (C) & P. 100 \\
\hline $\begin{array}{l}\text { Maïs } \ldots \ldots \ldots \ldots \ldots \\
\text { Tourteau de soja }(45 \% \\
\text { de protéines) } \ldots \ldots \ldots \ldots \\
\text { Farine de poisson (Nor- } \\
\text { vège) } \ldots \ldots \ldots \ldots \ldots \ldots \\
\text { Complément minéral } \\
\text { et vitaminique }{ }^{\left({ }^{2}\right)} \ldots\end{array}$ & $\begin{array}{r}28 \\
4\end{array}$ & $\mid \begin{array}{l}\text { Maïs } \ldots \ldots \ldots \ldots \ldots \\
\text { Avoine } \ldots \ldots \ldots \ldots \ldots \ldots \\
\text { Tourteau de soja }(45 \% \% \\
\text { de protéines) } \ldots \ldots \ldots \ldots \\
\left.\text { Complément minéral }{ }^{3}\right) \\
\text { et vitaminique }\left({ }^{2}\right) \ldots\end{array}$ & $\begin{array}{l}60 \\
15 \\
20 \\
5\end{array}$ & $\begin{array}{l}\text { Maĩs } \ldots \ldots \ldots \ldots \ldots \\
\text { Avoine } \ldots \ldots \ldots \ldots\end{array}$ & $\begin{array}{l}55 \\
45\end{array}$ \\
\hline
\end{tabular}

(1) Complément minéral (p. 100 du régime) : phosphate bicalcique 0,8 ; carbonate de calcium 1,7; sel 0,5 ; oligo-éléments (mélange commercial) 0,5 .

(2) Complément vitaminique (en g pour $100 \mathrm{~kg}$ de régime) : microvitamine A 16 ; microvitamine $\mathrm{D}_{3} 1$; riboflavine 0,2 ; vitamine $K_{3} 1$; vitamine $B_{12}(1 / 10000) 10$; vitamine $E 11$; pantothénate de calcium 0,2 ; acide nicotinique 0,5 ; choline (25 p. 100) 380 ; sulfate de manganèse 30 ; DL- méthionine 30 ; BHT 12,5; avoine broyée, q.s.p. 500 .

(3) Complément minéral (p. 100 du régime) : phosphate bicalcique 2 ; carbonate de calcium 1,5; sel 0,5 ; oligo-éléments (mélange commercial) 0,5 .

\section{Mesures}

Pour connaître les performances des animaux, leur poids et leur consommation alimentaire sont enregistrés toutes les deux semaines au début de l'expérience, puis chaque semaine à partir du $5^{6}$ jour. A la fin, nous estimons l'état d'engraissement des animaux en prélevant et pesant les graisses abdominales sur un échantillon représentatif de chaque lot. En effet, selon DELPECH et RICARD (1965), il existe chez le poulet une relation étroite entre les graisses abdominales et les lipides corporels.

\section{RÉSULTATS}

\section{Croissance, consommation et efficacité alimentaires}

La figure I représente l'évolution pondérale des animaux et schématise les plans d'alimentation. L'effet des diverses restrictions apparaît nettement et en fin d'expérience le poids moyen de chaque lot avoisine le poids de $2 \mathrm{~kg}$ qui avait été fixé. Pour le lot $D$, le plan d'alimentation se décompose ainsi : de o à 6 semaines les poulets reçoivent le régime de démarrage, de 6 à 9 semaines le régime de finition et enfin le mélange de céréales. Le lot $F$ reçoit jusqu'à ro semaines, le régime de finition puis pendant les $\mathrm{I}_{4}$ derniers jours le mélange de céréales. Les animaux du lot $3 \mathrm{C}-4 \mathrm{D}$ ont un développement pondéral lent mais leur croissance apparente est régulière et linéaire de 6 à $\mathrm{I} 2$ semaines (33 $\mathrm{g} / \mathrm{jour})$. Aussi, la périodicité dans la nature des apports alimentaires est-elle maintenue tout au long de l'expérience. 
Par contre, dans le lot 7 C-7 D, le ralentissement est trop important et la restriction est levée à Io semaines, date à partir de laquelle les sujets reçoivent uniquement le régime de démarrage. A cet âge, les animaux présentent encore un potentiel de

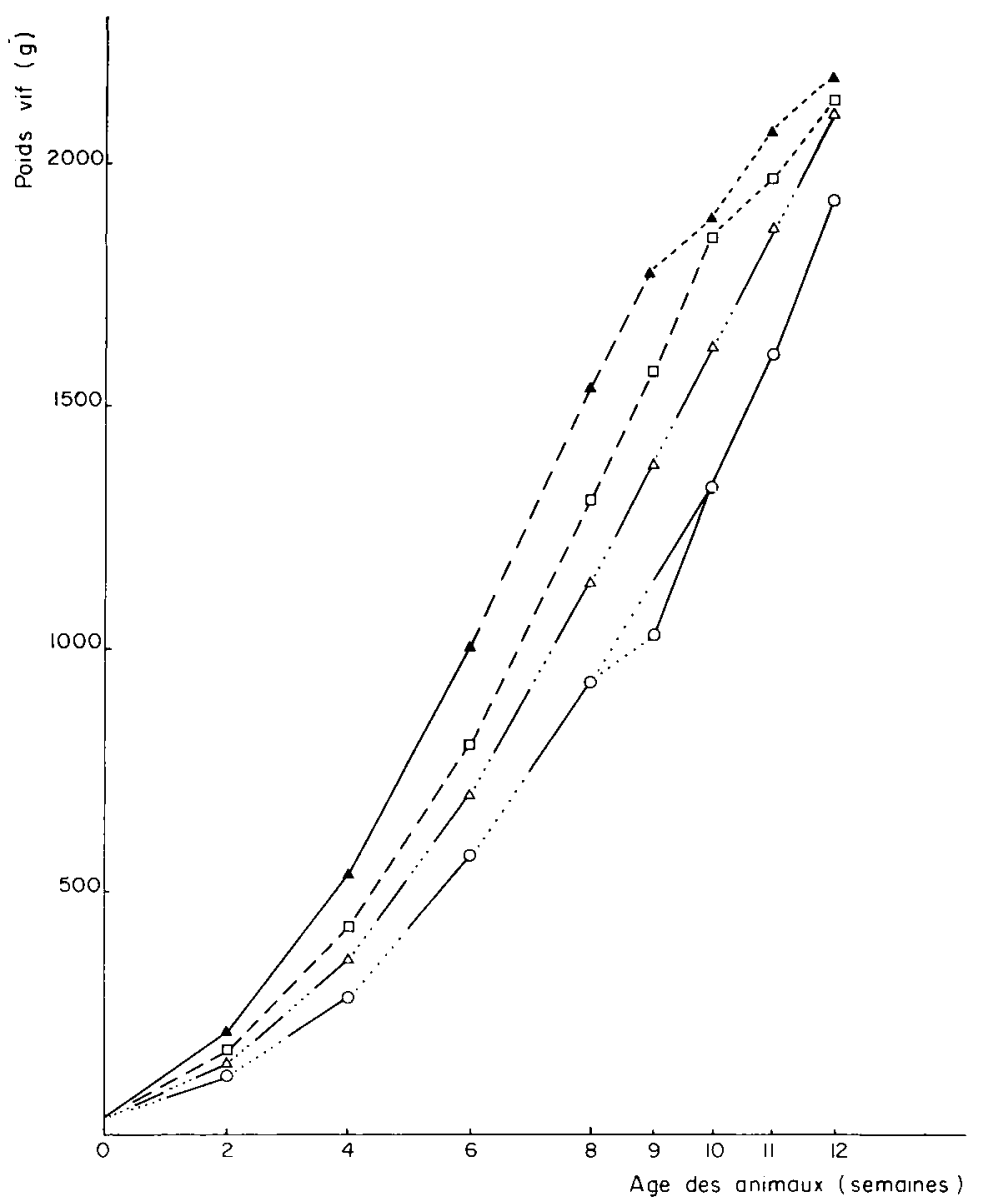

FIG. 1. - Évolution du poids vif dans les différents lots au cours de l'expérience

$$
\begin{aligned}
& \text { - } \operatorname{lot} \mathrm{D} \\
& \text { } \operatorname{lot} \mathrm{F} \\
& \triangle \quad \text { lot } 3 \mathrm{C}_{-4} \mathrm{D} \\
& \text { - lot } 7 \text { C-7 D } \\
& \begin{array}{l}
{ }^{\circ} \text { Régime démarrage (D) } \\
\text { Régime finition (F) } \\
\text { Régime céréales (C) }
\end{array}
\end{aligned}
$$

Dans le lot $7 \mathrm{C}-7 \mathrm{D}$, le point à 9 semaines est obtenu après l'alimentation en céréales. Pour la période 8 à Io semaines, nous avons fait figurer : la croissance réelle et comme pour les autres périodes la croissance virtuelle.

croissance élevé. Ils réalisent quotidiennement un gain de poids de 42 grammes, ce qui leur permet de compenser en deux semaines la majeure partie de leur retard.

Le tableau 2 consigne les performances globales de croissance. Nous enregistrons peu de variations dans le poids vif des animaux. Seul, le lot 7 C-7 D apparait 


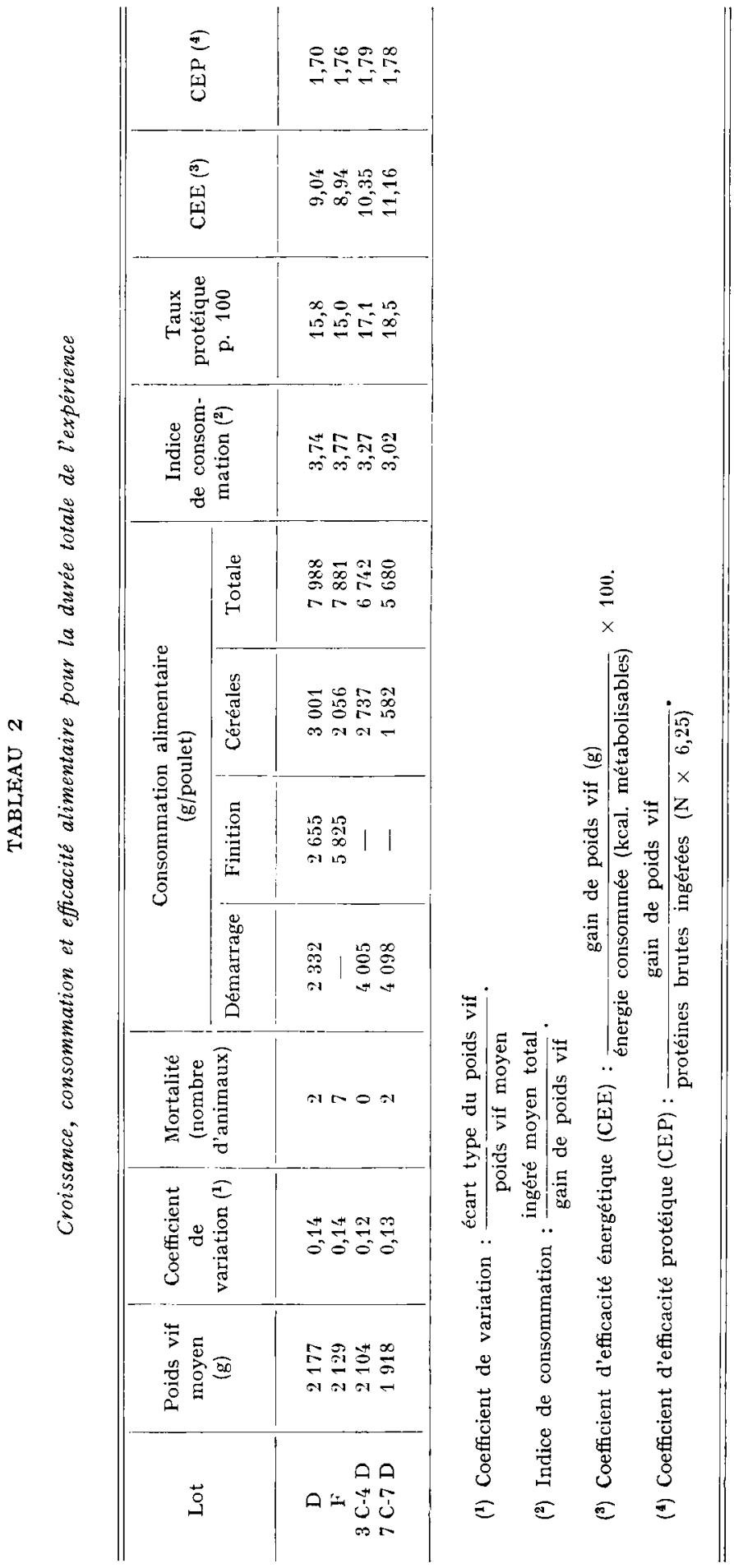


significativement plus léger $(\mathrm{P}<\mathrm{O}, \mathrm{OI})$. En outre, il n'existe aucune différence entre les coefficients de variation. Les diverses restrictions appliquées n'affectent donc pas l'homogénéité des lots.

La mortalité des différents lots ne varie pas significativement. Nous constatons toutefois qu'elle est plus élevée chez les animaux qui ont reçu dès la naissance le régime de finition.

D'après la consommation alimentaire totale, les animaux se classent selon la même hiérarchie que pour leur poids vif. Toutefois, nous observons une grande amplitude de variation. Plus la vitesse de croissance initiale est élevée, plus la consommation alimentaire croît. Ainsi les sujets des lots $3 \mathrm{C}-4 \mathrm{D}$ et $7 \mathrm{C}-7 \mathrm{D}$, ralentis dès leur naissance, ingèrent respectivement I 200 et 2300 grammes de moins que $\mathrm{D}$ et $\mathrm{F}$. Dans les deux cas, la consommation du régime démarrage est plus faible que la somme des ingérés démarrage et finition des lots $\mathrm{D}$ et $\mathrm{F}$. En outre pour le lot $7 \mathrm{C}-7 \mathrm{D}$, nous observons également une consommation réduite de céréales, car leur distribution est interrompue à l'âge de ro semaines.

Comme le poids final des poulets est du même ordre dans tous les lots, les différences de consommation se retrouvent pour les mesures de l'efficacité alimentaire. L'indice de consommation pour l'ensemble des 12 semaines, identique pour $\mathrm{D}$ et $\mathrm{F}$, diminue considérablement pour les lots soumis à l'alternance alimentaire. L'indice le plus bas correspond à la période de $\mathrm{I}_{4}$ jours (lot $7 \mathrm{C}-7 \mathrm{D}$ ). Le calcul des efficacités énergétiques et azotées nous donne une indication sur l'économie des nutriments réalisée par l'alimentation discontinue. L'efficacité énergétique mesurée par le rapport entre le gain de poids et 1'ingéré exprimé en calories métabolisables nous montre une amélioration significative par rapport aux lots $D$ et $F(P<0,01)$. Avec l'alternance 7 jours de céréales, 7 jours de démarrage, le coefficient d'efficacité énergétique est significativement plus élevé que pour le rythme $3 \mathrm{C}-4 \mathrm{D}(\mathrm{P}<0,0 \mathrm{I})$. En ce qui concerne le coefficient d'efficacité protéique, la progression est différente car le lot $\mathrm{D}$ est significativement différent des trois autres $(\mathrm{P}<0,05)$ et les deux alternances sont équivalentes. Ceci peut également être exprimé en calculant le taux protéique moyen qui résulte de la consommation totale d'aliment. Nous constatons alors que la proportion des protéines dans 1'aliment est plus élevée chez les lots soumis à l'alternance alimentaire (tabl. 2).

Ainsi, lorsqu'il est nécessaire de freiner la croissance des animaux, l'avantage de la restriction intermittente apparaît nettement. Par rapport à un développement entravé tardivement (lot D) ou régulièrement et modérément (lot $F$ ), la distribution périodique d'un mélange de céréales permet pour un même poids final de réduire la consommation alimentaire totale et d'accroître 1'efficacité alimentaire, cette amélioration portant sur l'utilisation à la fois de l'énergie et des protéines de la ration. Notons enfin que la distribution intermittente des céréales n'en augmente pas la consommation totale; elle n'entraîne pas non plus une réduction de la part des protéines dans les ingestats de l'ensemble de la période expérimentale.

\section{État d'engraissement}

Les graisses abdominales ont été utilisées comme indice d'adiposité. Lorsqu'on exprime celles-ci en valeur absolue ou en p. Ioo du poids vif (tab1. 3), les animaux restreints périodiquement se révèlent considérablement plus maigres $(\mathrm{P}<\mathrm{o}, \mathrm{OI})$. 
Toutefois, seul le lot 7 C- 7 D paraît, à 1'abattage, manquer de graisses de couverture. Parmi les deux autres groupes $D$ et $F$, le lot D qui présente la croissance la plus rapide durant les premières semaines, est significativement plus gras $(P<0,05)$. Ainsi les variations d'adiposité des animaux entre les lots expérimentaux reflètent celles de l'efficacité alimentaire et notamment de l'efficacité énergétique.

TABLEAU 3

État d'engraissement des animaux (1)

\begin{tabular}{c|c|c|c|c|c}
\hline & Lot & $\mathrm{D}$ & $\mathrm{F}$ & $3 \mathrm{C}-4 \mathrm{D}$ & $7 \mathrm{C}-7 \mathrm{D}$ \\
\hline $\begin{array}{c}\text { Graisses } \\
\text { abdominales }\end{array}$ & En valeur absolue $(\mathrm{g})$ & $\mathbf{7 0 , 1 9 \pm 5 , 8 5}$ & $53,35 \pm 6,58$ & $32,79 \pm 3,75$ & $22,52 \pm 2,82$ \\
\hline En p. 100 du poids vif & $3,13 \pm 0,22$ & $2,42 \pm 0,27$ & $1,48 \pm 0,16$ & $1,10 \pm 0,12$ \\
\hline
\end{tabular}

(1) Nous donnons la moyenne et l'écart-type de la moyenne pour $n=26$ à 28 animaux.

\section{Prix de revient de l'alimentation}

L'allongement de la durée d'élevage nécessaire à la production de poulets de qualité implique une augmentation du prix de revient. Comme l'ont montré HELBACKA et Combs (I959), la part de l'alimentation augmente de 60 à $66 \mathrm{p}$. Ioo quand 1'âge d'abattage s'accrôit de 8 à I2 semaines. Nous avons donc effectué un calcul comparatif du prix des aliments consommés dans les différents lots (tabl. 4).

TABLEAU 4

Résultats économiques: coût de l'alimentation $\left({ }^{\mathbf{1}}\right)$

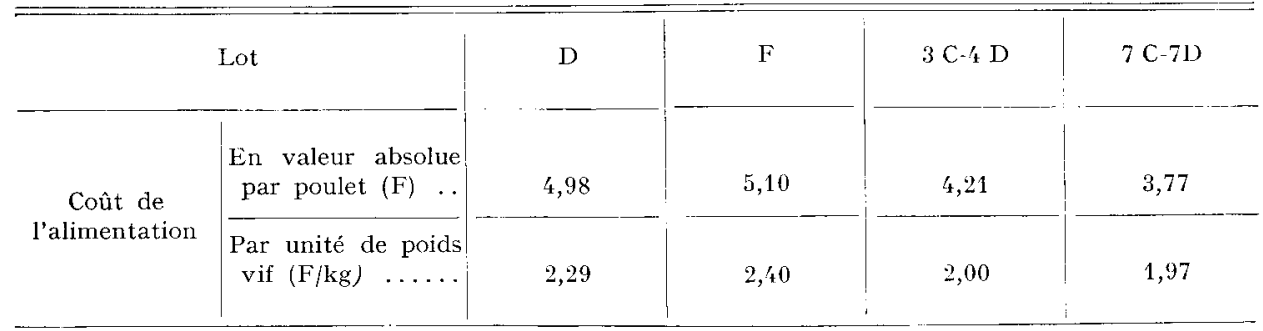

(1) Pour faire ces calculs, nous avons utilisé les prix suivants (en francs $/ \mathrm{kg}$ ) :

$\begin{array}{ll}\text { Régime démarrage.... } & 0,75 \\ \text { Régime finition ...... } & 0,72 \\ \text { Maïs .............. } & 0,46 \\ \text { Avoine .......... } & 0,41\end{array}$


Les valeurs que nous avons utilisées correspondent aux prix courants pratiqués dans le commerce des aliments du bétail au I er janvier I97I. Les résultats obtenus ainsi montrent que l'alternance alimentaire entraîne une économie relative hautement significative $(\mathrm{P}<\mathrm{o}, \mathrm{or})$, en valeur absolue aussi bien que par $\mathrm{kg}$ de poids vif. A l'inverse, le ralentissement modéré et continu imposé au lot $\mathrm{F}$ entraîne le coût alimentaire le plus élevé $(\mathrm{P}<\mathrm{O}, \mathrm{OI})$. Il est certain que ces calculs se prêtent à la critique car nous nous sommes placés dans des conditions arbitraires de prix des aliments. Cependant, les économies de consommation portant principalement sur les régimes complexes, démarrage et finition, nous estimons que le classement des coûts de l'aliment demeure lorsque les variations de prix relatives des régimes complets par rapport aux céréales sont conservées.

\section{DISCUSSION ET CONCI,USION}

Pour ne pas surcharger cette étude nous tiendrons compte des seules considérations zootechniques, l'effet des discontinuités alimentaires ayant été discuté par ailleurs tant chez le poulet (Simon et $a i$, J968; Simon et Blum, sous presse) que chez le rat (Morin-Jomain, Ig68).

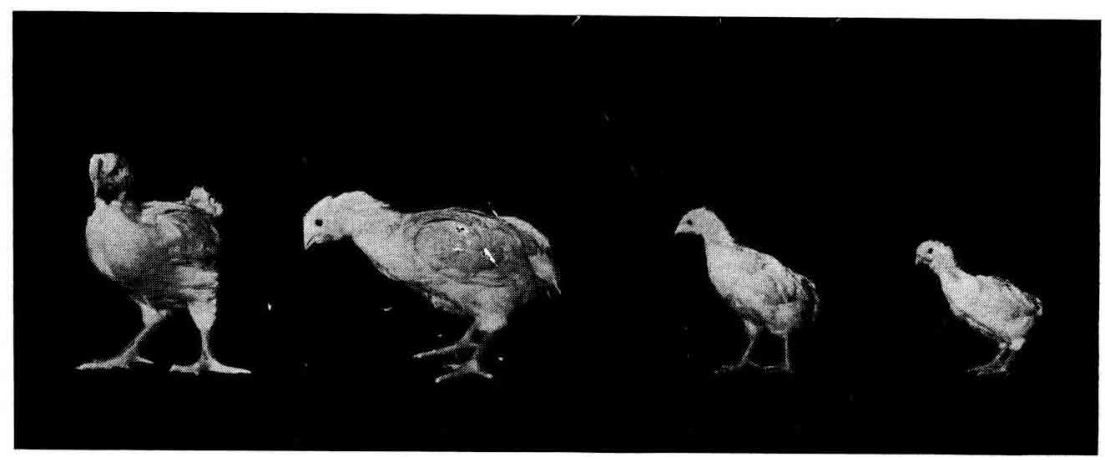

$$
\begin{array}{llll} 
& \text { FIG. 2. }- \text { Emplumement des poulets à } 45 \text { jours } \\
\text { Lot } \mathrm{D} & \text { Lot } \mathrm{F} & \text { Lot } 3 \mathrm{C}_{-4} \mathrm{D} & \text { Lot } 7 \mathrm{C}-7 \mathrm{D}
\end{array}
$$

On observe un retard dans l'emplumement au fur et à mesure que la restriction alimentaire est plus importante dans les premières semaines de la vie postnatale. Ainsi les poulets des lots $7 \mathrm{C}-7 \mathrm{D}$ et 3 C-4 D possèdent encore des duvets sur leur cou alors que les plumes sont en train de les remplacer chez ceux des lots $F$ et surtout $D$.

Les restrictions alimentaires mises en ceuvre dans cette étude permettent d'aboutir à un poids sensiblement identique à I2 semaines; d'après la figure $I$, il est probable que les animaux plus légers du lot $7 \mathrm{C}-7 \mathrm{D}$ auraient atteint un poids supérieur à $2 \mathrm{~kg}$ si nous avions écourté la distribution des céréales durant la neuvième semaine. Mais ces restrictions entravent de différentes façons le développement pondéral des animaux. Aussi les performances diffèrent-elles considérablement selon les lots.

Sur la figure 2, nous observons qu'outre leur effet sur le poids vif, les plans 
d'alimentation ont influencé le développement de chaque tissu des animaux : par exemple les duvets sont encore présents sur le cou des oiseaux soumis à l'alimentation alternée alors qu'ils ont disparu chez les poulets des lots $F$ et $D$ âgés de 45 jours. Ces animaux apparaissent donc physiologiquement plus jeunes.

La méthode traditionnelle (lot D) de ralentissement de la croissance du poulet s'accompagne d'une élévation importante de la consommation alimentaire et d'un abaissement parallèle de l'efficacité. Il en est de même des animaux qui reçoivent dès la naissance un régime moins riche (lot $F$ ). En outre, les céréales distribuées en fin d'expérience provoquent un engraissement considérable. Ceci est conforme aux résultats de HARSHAW (I938), qui montrent que la distribution de céréales pendant deux semaines à des poulets de différents âges entraîne un engraissement d'autant plus marqué que les animaux sont plus âgés.

A l'inverse, l'alimentation alternée s'avère bénéfique pour tous les critères mesurés : performances zootechniques, adiposité et coût de 1'alimentation. Cette technique d'alimentation permet de réaliser une restriction qualitative. Le tableau 5

TABLEAU 5

Evolution du taux protidique moyen (p. Iоo)

calculé d'après la consommation des animaux entraînés à l'alimentation alternée

\begin{tabular}{|c|c|c|c|c|c|c|}
\hline Age (semaines) & $0-2$ & $0-4$ & $0-6$ & $0-8$ & $0-10$ & $0-12$ \\
\hline $\begin{array}{lllll}\text { Lot } 3 & C-4 & D & \ldots \\
\text { Lot } & 7 & C-7 & D & \ldots\end{array}$ & $\begin{array}{l}19,3 \\
19,4\end{array}$ & $\begin{array}{l}17,6 \\
17,0\end{array}$ & $\begin{array}{l}17,6 \\
17,5\end{array}$ & $\begin{array}{l}17,8 \\
17,3\end{array}$ & $\begin{array}{l}17,2 \\
17,1\end{array}$ & $\begin{array}{l}17,1 \\
18,5\end{array}$ \\
\hline
\end{tabular}

rapporte 1'évolution du taux protidique moyen calculé d'après la consommation des deux régimes. En dehors des deux premières semaines aux cours desquelles les animaux consomment peu de céréales et des deux dernières pour le lot $7 \mathrm{C}-7 \mathrm{D}$ disposant alors du régime démarrage, le taux protidique s'établit à I7 $\mathrm{p}$. Ioo pendant toute l'expérience. Selon toute vraisemblance la distribution en continu d'un tel aliment ne permettrait pas d'obtenir le même résultat. En effet, la comparaison des différents lots à 6 semaines d'âge (tabl. 6) montre que pour un taux protidique intermédiaire, les animaux soumis à l'alimentation alternée présentent le développement pondéral et les consommations alimentaires les plus faibles. Dans les deux lots alternés, l'indice de consommation est plus élevé que dans le lot $\mathrm{D}$ mais plus bas que dans le lot F. Assurant une meilleure efficacité alimentaire et ralentissant davantage la croissance qu'une restriction protidique globale continue, l'alimentation alternée réalise simultanément un rationnement qualitatif et un rationnement quantitatif. La déficience en lysine des protéines des céréales peut jouer un rôle dans la réduction des ingérés (SINGSEN et al., r965). Par ailleurs, les animaux ralentis ont un potentiel de croissance exacerbé de telle sorte que de 9 à I2 semaines on enregistre dans le lot $7 \mathrm{C}-7 \mathrm{D}$ un gain de poids de $903 \mathrm{~g}$ (43 g/jour) et une efficacité alimentaire de 2,84 . En définitive, la nette supériorité rencontrée en alimentation alternée sur l'ensemble de l'expérience trouve son origine dans la précocité de la 
restriction et dans son caractère intermittent qui en fait une méthode de rationnement à la fois qualitatif et quantitatif qui tient compte des capacités d'adaptation aux restrictions alimentaires discontinues dont font preuve les poulets (SrMoN et al., Ig68; Simon et BLum, sous presse).

TABLEAU 6

Croissance, consommation et efficacité alimentaire des animaux à 6 semaines d'age

\begin{tabular}{|c|c|c|c|c|c|c|c|}
\hline \multirow{2}{*}{ Lot } & \multirow{2}{*}{$\begin{array}{l}\text { Poids vif } \\
\text { (g) }\end{array}$} & \multicolumn{4}{|c|}{ Consommation alimentaire } & \multirow{2}{*}{$\begin{array}{l}\text { Indice de } \\
\text { consommation }\end{array}$} & \multirow{2}{*}{$\begin{array}{c}\text { Taux } \\
\text { protéique }\end{array}$} \\
\hline & & Démarrage & Finition & Céréales & Totale & & \\
\hline D & 1018 & 2332 & 0 & 0 & 2332 & 2,38 & 21,6 \\
\hline F & 812 & 0 & 2263 & 0 & 2263 & 2,93 & 16,6 \\
\hline $3 \mathrm{C}-4 \mathrm{D}$ & 702 & 1137 & 0 & 606 & 1743 & 2,63 & 17,6 \\
\hline $7 \mathrm{C}-7 \mathrm{D}$ & 567 & $87 / 4$ & 0 & 505 & 1379 & 2,62 & 17,5 \\
\hline
\end{tabular}

L'alimentation alternée semble donc convenir parfaitement à la production de poulets de chair de qualité qui sont nécessairement âgés.

Nos résultats soulignent en outre l'importance primordiale du stade de développement où la restriction est appliquée. Lorsqu'un ralentissement de la croissance du poulet s'avère nécessaire, il doit être réalisé dès la naissance de l'animal.

\title{
Requ pour publication en janvier 1972.
}

\author{
SUMMARY
}

\section{FEED RESTRIC'TION METHODS FOR REGUI,ATING}

THE FIRST TWELVE, WEEKS OF GROWTH IN BROILER CHICKENS

Broiler chicken market includes two main types of products : "Industrial Chickens ", sacrified between 6 and 9 weeks and obtained with maximum growth rate and feed efficiency, and "High Quality Chickens ", slaughtered at 12 weeks at a live weight of about $2 \mathrm{~kg}$. The production of the second type of animals is expensive because of the forced reduction of their growth rates. Therefore, we looked for a low cost feeding method able to meet the requirement of gourmet consumers.

The trial was carried out with one day old Arbor Acres chicken. The aim was to obtain live weights of $2 \mathrm{~kg}$ at $\mathrm{I} 2$ weeks by using different techniques. The feed was always offered ad libitum. Three feeding schedules were compared. In the first case (group D), the animals, received successively : a starter diet from o to 6 weeks ( 2 r.6 p. Ioo protein), a finishing diet from 6 to 9 weeks (16.6 p. Ioo protein), and a mixture of cereals for the last three weeks. In the second one (group F), the animals were fed a finishing diet from birth and then a mixture of cereals from the age of Io weeks. In the third case, the animals received, from birth, the cereal mixture in alternation with the starter diet. Two periods have been studied : either 3 days with the cereal diet and then 4 days with the starter diet (group $3 \mathrm{C}-4 \mathrm{D}$ ) or 7 days with cereals and then 7 days with the starter diet (group $7 \mathrm{C}-7 \mathrm{D}$ ). 
As expected, the weights of the animals at slaughter were rather similar. However, for all criteria (feed intake and feed efficiency, fattening, and feeding costs), the distribution of the finishing diet from birth appeared to be the worst method, whereas periodical feed restriction was very favourable. The advantage of alternated feeding (cereals, starter diet) depended on several factors. Being applied from the young age, it resulted in reduction of expenditures for total maintenance. During the phases of repletion, the animals were fed rich diets ensuring maximum feed efficiency. Finally, it is known that the animals adapt themselves to discontinous feeding (Simon, Blum and JAcquot, rg68; Simon and Blum, in press). Consequently, this method can give very favourable results when used to produce high quality broiler chicken.

\section{RÉFÉRENCES BIBLIOGRAPHIQUES}

Delpech P., 1963. Les qualités jugées par l'éleveur, le conditionneur et le consommateur sont-elles compatibles? Ind. aliment. Anim., 143, 37-57.

Delpech P., 1968. Estimation et signification de quelques critères de la qualité du Poulet. Alimentation et la Vie, 56, 283-306.

DELPECH P., 1969. Le poulet, moderne, sa préparation, son aspect, sa saveur. Économie et Médecine animale, 10, 337-348.

Delpech P., Ricard F.-M., I965. Relation entre les dépôts adipeux viscéraux et les lipides corporels chez le Poulet. Ann. Zootech., 14, r8 I-I89.

Harshaw H. M., I938. The effect of fattening at different ages on the composition of Cockerels. Poult. Sci., 17, 163-169.

Helbacka N. V., Combs G. F., I959. Cost of producing broiler meat. Feedstuffs, 31 (46), 18-19.

Morin-Jomain M., 1968. influence des modalités alimentaires sur la croissance et la composition corporelle du rat blanc. Particularités de l'alimentation discontinue. Thèse Doctorat, Université de Paris.

Simon J., Blum J.-C., I972. Étude de l'influence de jeûnes périodiques sur la croissance, la glycémie, quelques caractéristiques du métabolisme hépatique et la composition corporelle du Poulet. Can. $J$. Physiol. Pharmacol. (sous presse).

Simon J., Blum J.-C., Jacouot R., ig68. Étude des effets de l'alimentation discontinue chez le Poulet : variations pondérales et composition corporelle. C. R. Acad. Sci. Paris, 267, série D, 20022004 .

Singsen E. P., Nagel J., Patrick S. G., Matterson L. D., ig65. The effect of a lysine deficiency on growth characteristics, age at sexual maturity and reproductive performance of meat type pullets. Poult. Sci., 44, 1467-1473. 